\title{
Dépôt pollinique actuel et végétation du sud du Bénin
}

\author{
Gbèkponhami Monique TOSSOU ${ }^{1 *}$, Hounnankpon YEDOMONHAN ${ }^{1}$, \\ Cossi Aristide ADOMOU ${ }^{1}$, Akpovi AKOEGNINOU ${ }^{1}$ et Koffi AKPAGANA ${ }^{2}$ \\ ${ }^{I}$ Département de Biologie végétale, Faculté des Sciences et Techniques, Université d'Abomey-Calavi, \\ 01 BP 4521, Cotonou, Bénin. \\ ${ }^{2}$ Laboratoire de Botanique et d'Ecologie Végétale, Faculté des Sciences, Université de Lomé, \\ BP 1515, Lomé, Togo. \\ *Auteur correspondant, E-mail : tossou@bj.refer.org / tossoumonique@yahoo.fr
}

\section{RESUME}

Les analyses polliniques de sédiments de surface, prélevés dans les différentes formations végétales qui constituent le paysage du sud du Bénin, sont réalisées en vue d'établir une relation entre la pluie pollinique et la végétation actuelle. Les pollens des espèces dominantes des principaux types de formations végétales sont identifiés, mais leur taux de représentativité pour chaque type de formation végétale est resté faible. La végétation locale n'est donc pas la plus représentée dans les spectres polliniques. Ainsi, la pluie pollinique enregistre aussi bien les données de la végétation locale que régionale. Le diagramme pollinique obtenu montre l'importance des pollens de Elaeis guineensis et des Poaceae qui sont partout quasi-présents, ce qui caractérise la dominance des formations ouvertes, résultant surtout de l'action anthropique dans le sud du Bénin.

(c) 2012 International Formulae Group. All rights reserved.

Mots clés: Pluie pollinique, pollen, formations végétales.

\section{INTRODUCTION}

La pluie pollinique qui est l'ensemble des pollens et des spores déposés en un même lieu, représente plus ou moins fidèlement la végétation, ou tout au moins, il existe une relation étroite entre elle et la végétation (Reille et Pons, 1990). L'étude des dépôts polliniques actuels permet une interprétation des diagrammes polliniques fossiles (Lézine et Edorh, 1991). Dans ce cadre, des études sur la pluie pollinique actuelle ont été effectuées, tant dans le nord de l'Afrique, au Sahara (Cour, 1974; Cour et Duzer, 1976) qu'en Afrique intertropicale (Maley, 1972; Ybert, 1975; Edorh, 1986 ; Lézine et Edorh, 1991; Vincens et al., 2000 ; Elenga et al., 2000).
Elles ont montré qu'il existe de nombreuses variations liées aux particularités géographiques et topographiques de chaque site étudié. Dans le Sahara, la pluie pollinique est dominée par l'apport lointain tandis que dans la zone intertropicale, c'est la végétation locale qui transparaît dans la sédimentation pollinique actuelle. La production et le type de dispersion des pollens donnent lieu à un enregistrement aussi bien local que régional (Maley, 1972 ; Reynaud-Farrera, 1995 ; Elenga et al., 1996 ; Vincent et al., 2000). En Afrique occidentale, les études sur la pluie pollinique actuelle ont été effectuées en Côte d'Ivoire (Ybert, 1975), au Sénégal (Lézine et Edorh, 1991) et au Togo (Edorh et Afidégnon, 
2008). Les premières études du genre ont eu lieu au Bénin dans le cadre du Projet «Dahomey-Gap» dont l'objectif est de donner une meilleure interprétation des spectres polliniques fossiles holocènes pour comprendre l'histoire du Dahomey-Gap. En effet, le «Dahomey-Gap» est la coupure entre les deux massifs forestiers guinéen et congolais où les savanes arrivent jusqu'à la côte (Jenik, 1994). Le but du présent travail est d'apprécier la représentativité des dépôts polliniques actuels sous les différents types de formation végétale du Sud du Bénin.

\section{MATERIEL ET METHODES Milieu d'étude}

La zone d'étude est la partie sud du Bénin située entre $6^{\circ} 15^{\prime}$ et $7^{\circ} 00^{\prime}$ de latitude Nord et $1^{\circ} 45^{\prime}$ et $2^{\circ} 45^{\prime}$ de longitude Est (Figure 1). Sa géologie est caractérisée par des formations sédimentaires variées (Rabier, 1978). Le relief est marqué par des plateaux et des plaines découpés par des cours d'eau dont les plus importants sont l'Ouémé et le Mono, des lacs et des lagunes. Les sols drainés sont en majorité de type ferrallitique. Ils sont profonds et comportent en leur sein des sols hydromorphes et des vertisols. Le climat est de type subéquatorial avec la pluviométrie comprise entre 737,6 mm à l'Ouest et 1458,9 $\mathrm{mm}$ à l'Est (Akoègninou, 2004). La végétation est caractérisée par diverses formations végétales, à savoir : la forêt dense humide semi-décidue à Celtis, la forêt claire à Lophira lanceolata, les fourrés secondaires, la forêt marécageuse, la mangrove, la savane à Mitragyna inermis, les prairies marécageuses ou inondables et les plantations dont les cocoteraies, les palmeraies et la plantation à Acacia spp. (Akoègninou, 2004).

\section{Méthode d'étude}

La méthode utilisée a consisté à prélever la partie superficielle du sol comme l'ont précédemment fait Bonnefille (1972), Salgado-Labouriau (1979), Lézine (1981) et Edorh (1986). Les échantillons ont été collectés dans six formations végétales (Tableau 1). Pour chaque échantillon, la partie superficielle du sol de $0-5 \mathrm{~cm}$ de profondeur a été prélevée et conservée dans un sachet plastique hermétiquement fermé.

Un inventaire botanique a été effectué dans un rayon de $50 \mathrm{~m}$ autour du lieu de prélèvement. Cette distance a été adoptée en s'inspirant des considérations de Heim (1970) qui distingue apport local $(0$ à $50 \mathrm{~m})$, apport extra-local $(50 \mathrm{~m}$ à $500 \mathrm{~m})$, apport régional $(500 \mathrm{~m}$ à $10 \mathrm{~km}$ ) et apport lointain (plus de 10 $\mathrm{km})$.

Au laboratoire, chaque échantillon a été passé à travers un tamis dont les mailles sont de $200 \mu \mathrm{m}$ de diamètre afin d'éliminer les grosses particules. Dix (10) grammes de la partie fine ont été traités selon la méthode classique de Faegri et Iversen (1989). Les échantillons ont ensuite subi l'acétolyse et après plusieurs centrifugations et rinçages à l'eau distillée, le culot a été dilué dans de l'huile de silicone et une toute petite quantité a été montée entre lame et lamelle pour l'observation et l'analyse au microscope Olympus®.

Les pollens ont été comptés et identifiés aux grossissements $(\times 200)$ et $(\times$ 400). Le comptage a consisté à un balayage complet de toute la surface de la lamelle. Les identifications ont été effectuées par comparaison avec les collections de lames de référence du Laboratoire de Botanique et d'Ecologie végétale de l'Université d'Abomey-Calavi et les illustrations des ouvrages sur les pollens de Caratini et Guinet (1974), de Ybert (1979) et de Sowunmi (1973, 1995). Ces identifications botaniques des pollens ont été faites soit seulement au niveau de la famille, soit du genre et/ou de l'espèce. Les nomenclatures utilisées sont celles de la Flore Analytique du Bénin (Akoègninou et al., 2006).

Les résultats de l'analyse pollinique ont été présentés sous forme d'un diagramme pollinique qui a été construit à l'aide du programme TGView (Grimm, 2004). Ils ont aussi servi à établir la matrice échantillons de surface-taxons qui a été soumise à la classification hiérarchique à l'aide du logiciel CAP (Community Analysis Package) en vue d'identifier des groupes d'échantillons de surface sur la base de leur ressemblance pollinique. 
Tableau 1: Caractéristiques géographiques des sites, types de végétation et nombre de prélèvements.

\begin{tabular}{|c|c|c|}
\hline Localités & Coordonnées géographiques & $\begin{array}{c}\text { Nombre de } \\
\text { Prélèvements }\end{array}$ \\
\hline \multicolumn{3}{|l|}{ Forêt dense semi-décidue } \\
\hline Adjrahounvè (Ad1-Ad8) & $6^{\circ} 25^{\prime} 36^{\prime \prime} \mathrm{N}-2^{\circ} 12^{\prime} 38^{\prime \prime} \mathrm{E}$ & 8 \\
\hline Niaouli (Ni1-Ni3) & $6^{\circ} 44^{\prime} 38^{\prime \prime} \mathrm{N}-2^{\circ} 08^{\prime} 25^{\prime \prime} \mathrm{E}$ & 3 \\
\hline Zinvié (Z1-Z2) & $6^{\circ} 37^{\prime} 58^{\prime \prime} \mathrm{N}-2^{\circ} 21^{\prime} 48^{\prime \prime} \mathrm{E}$ & 2 \\
\hline \multicolumn{3}{|c|}{ Plantation à Acacia auriculiformis } \\
\hline Ahozon (Ah1-Ah3) & & 3 \\
\hline \multicolumn{3}{|l|}{ Forêt inondable } \\
\hline Ahozon (Ah4-Ah13) & $6^{\circ} 22^{\prime} 96^{\prime} \mathrm{N}-2^{\circ} 09^{\prime} 52^{\prime \prime} \mathrm{E}$ & 10 \\
\hline \multicolumn{3}{|l|}{ Mangrove } \\
\hline Ouidah (Ou1-Ou4) & $6^{\circ} 20^{\prime} 35^{\prime \prime} \mathrm{N}-2^{\circ} 05^{\prime} 22^{\prime \prime} \mathrm{E}$ & 4 \\
\hline Agatogbo (Ag1-Ag2) & $6^{\circ} 24^{\prime} 15^{\prime \prime} \mathrm{N}-1^{\circ} 56^{\prime} 06^{\prime \prime} \mathrm{E}$ & 2 \\
\hline \multicolumn{3}{|l|}{ Forêt marécageuse } \\
\hline Dogbocomey (Do1) & $6^{\circ} 32^{\prime} 22^{\prime \prime} \mathrm{N}-2^{\circ} 22^{\prime} 20^{\prime \prime} \mathrm{E}$ & 1 \\
\hline Adjavié (AJ1) & $6^{\circ} 34^{\prime} 15^{\prime \prime} \mathrm{N}-2^{\circ} 22^{\prime} 20^{\prime \prime} \mathrm{E}$ & 1 \\
\hline Goho (Go1-Go2) & $6^{\circ} 26^{\prime} 35^{\prime \prime} \mathrm{N}-2^{\circ} 34^{\prime} 45^{\prime \prime} \mathrm{E}$ & 2 \\
\hline \multicolumn{3}{|l|}{ Prairie marécageuse } \\
\hline Kraké (Kr1-Kr2) & $6^{\circ} 23^{\prime} 29^{\prime \prime} \mathrm{N}-2^{\circ} 40^{\prime} 42^{\prime \prime} \mathrm{E}$ & 2 \\
\hline
\end{tabular}

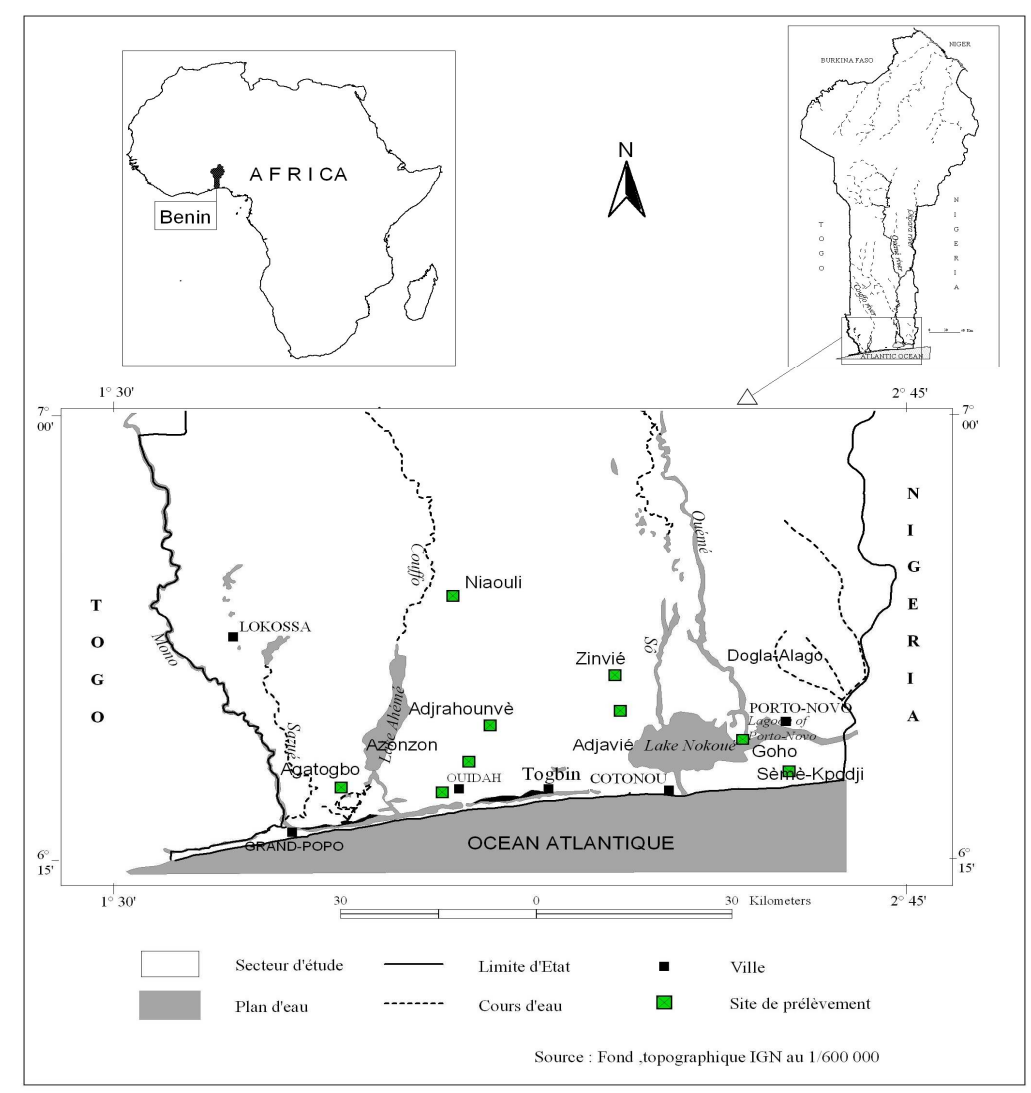

Figure 1: Localisation des sites de prélèvement des échantillons de surface. 


\section{RESULTATS ET DISCUSSION}

L'inventaire floristique a permis de recenser 237 espèces dont 82 dans la forêt dense humide semi-décidue, 45 dans la plantation à Acacia auriculiformis, 70 dans la forêt inondable, 50 dans la mangrove, 99 dans la forêt marécageuse et 22 dans la prairie marécageuse (Tableau 2).

Dans les 38 échantillons de sol analysés, 126 taxons sont identifiés (Tableau $2)$; ils se regroupent en 65 familles. La somme pollinique varie de 11 à 2500 pollens par échantillon avec une moyenne de 435 pollens par échantillon. Le diagramme pollinique (Figure 2) montre les taxons dont les pollens sont les plus abondants et qui sont indicateurs pour l'interprétation des données. Six (06) zones polliniques sont distinguées. Elles reflètent plus ou moins les différentes formations végétales prospectées.

\section{Forêt dense humide semi-décidue Inventaire floristique}

Certains des îlots sont dégradés mais leur physionomie est généralement marquée par quatre strates. La liste des 82 espèces inventoriées est présentée dans le Tableau 2. Les plus abondantes sont: Antiaris africana, Cola gigantea, Chassalia kolly, Pentachletra macrophylla, Monodora tenuifolia, Albizia glaberrima, etc.

\section{Spectre pollinique}

De l'analyse pollinique des treize prélèvements d'échantillons, 45 taxons sont identifiés (Tableau 2).

Les pourcentages de taxons d'herbacées, d'arbres et arbustes varient suivant les sites. Ils se situent entre $20 \%$ et $50 \%$ pour les herbacées et $50 \%$ et $80 \%$ pour les arbres et arbustes, soit en moyenne 36,3\% d'herbacées et $62,9 \%$ d'arbres et arbustes. Ce taux des arbres et arbustes indique qu'il s'agit d'une formation forestière mais il reste inférieur au taux de $70 \%$ proposé par Lebamba et al. (2008) dans les forêts du Gabon et du Cameroun. Mais selon Elenga (1992), Reynaud-Farrera (1995) et Elenga et al. (2000) dans les forêts denses, les pollens des taxons arborés sont sous-représentés ou absents des diagrammes polliniques à cause de la pollinisation entomophile de nombreux taxons de forêt. Les taux des Cyperaceae $(9,9 \%)$ et des Poaceae (9\%), taxons indicateurs de milieux ouverts (Vincens et al., 2000), sont relativement élevés. Ils sont supérieurs au taux de $2 \%$ enregistrés par Lebamba et al. (2008); ceci expliquerait l'état de dégradation de certains îlots de forêt et du paysage environnant souvent anthropisé. Selon Akoègninou (2004) et Akoègninou et al., (2006), la végétation du Sud-Bénin est caractérisée par son extrême émiettement marqué par la cohabitation d'îlots de forêt, de champs, de jachères, de fourrés et de savanes.

Dans le spectre pollinique, seulement $22 \%$ des taxons inventoriés dans les forêts sont aussi identifiés dans les échantillons de surface. Ce faible taux d'espèces inventoriées et identifiées dénote d'une faible représentativité des dépôts polliniques comparativement au taux préconisé par Reille et Pons (1990) qui est de $80 \%$. Mais les taxons tels que Celtis milbraedii, Adenia lobata, Pycnanthus angolensis, Cola gigantea, Drypetes floribunda et Dialium guineense, etc. caractéristiques des forêts denses semidécidues du sud du Bénin, se retrouvent dans le spectre pollinique en des proportions bien faibles. Le genre Celtis, selon Letouzey (1968) est un bon marqueur des forêts denses humides semi-décidues. De plus, selon Akoègninou (1984, 2004), Akpagana (1989), les espèces du genre Celtis, font partie des espèces les plus importantes de la forêt dense humide semi-décidue du Sud du Bénin, qualifiée de forêt à Celtis. Antiaris toxicaria très fréquente dans le paysage n'est pas identifié dans les échantillons. Cela peut être dû à la rareté de la floraison chez l'espèce et des Moraceae en général (Tossou, 2002). Les taxons allochtones ou seulement identifiés sont à un taux de $71,4 \%$. Ils comprennent des taxons de fourrés (Alchornea cordifolia, Chassalia kolly, Cnestis ferruginea, etc.), de 
mangrove (Rhizophora racemosa, Acrostichum aureum), de plantation (Acacia auriculiformis, Irvingia gabonensis, Pinus caraibaea) et des rudérales (Scoparia dulcis, Sida acuta, Hyptis suaveolens, Desmodium ramossisimum, etc.).

\section{Plantation à Acacia auriculiformis Inventaire floristique}

La plantation à Acacia auriculiformis, couvrant plusieurs hectares de superficie, est une formation monospécifique d'arbres de plus de dix ans d'âge dont la hauteur culmine vers $15 \mathrm{~m}$. Au total, 45 espèces sont inventoriées (Tableau 2). Dans le sous-bois, sont recensés des arbustes et des herbacées dont les plus fréquents sont: Adenia lobata, Chassalia kolly, Olax subscorpioidea, Rytiginia umbellulata, Agelaea pentagyna, Uvaria chamae, Diospyros tricolor. Cette plantation est bordée à l'Ouest par une forêt dense humide semi-décidue et au Nord par une forêt inondable.

\section{Spectre pollinique}

A l'analyse des 3 échantillons, 48 taxons sont identifiés (Tableau 2). Les pourcentages de taxons d'herbacées, d'arbres et arbustes varient suivant les sites. Ils se situent entre $17,2 \%$ et $35,3 \%$ pour les herbacées et $64,7 \%$ et $82,8 \%$ pour les arbres et arbustes, soit en moyenne $28,6 \%$ d'herbacées et $71,4 \%$ d'arbres et d'arbustes. Acacia auriculiformis (9,8\%), le taxon de reboisement et qui a une floraison ininterrompue, vient en troisième position après Adenia lobata $(43,3 \%)$ et Elaeis guineensis (11,5\%). En considérant l'inventaire floristique et l'analyse pollinique, on note que $31,1 \%$ des espèces inventoriées sont identifiées et que $70,8 \%$ de taxons sont seulement identifiées. Ce taux élevé d'allochtones indique un apport pollinique extra local ou régional comme en témoignent les taxons comme Talinum triangulare, Combretum indicum, Cyperaceae et Poaceae relevant des fourrés et de la forêt inondable adjacents à la plantation et Rhizophora racemosa et Acrostichum aureum, provenant de la mangrove située à plus de $20 \mathrm{~km}$ à vol d'oiseau. En effet, la présence des pollens de Rhizophora racemosa est due au caractère anémophile de ses pollens (Lézine, 1996).

\section{Forêt inondable Inventaire floristique}

La forêt inondable est située à Ahozon non loin de la plantation à Acacia auriculiformis au bord du lac Toho. Au total, 69 espèces sont recensées (Tableau 2). Les plus fréquentes sont: Pycnanthus angolense, Macaranga heudoletii, Parinari robusta, Cleistopholis patens, Symphonia globulifera, Chrysobalanus icaco var. ellipticus, Xylopia aethiopica, etc.

\section{Spectre pollinique}

A l'analyse pollinique des 10 échantillons, 96 taxons sont identifiés (Tableau 2). Les taux de taxons d'herbacées, d'arbres et arbustes varient d'un échantillon à un autre. Ils se situent entre $20 \%$ et $36,8 \%$ pour les herbacées et $63,2 \%$ et $80 \%$ pour les arbres et arbustes, soit en moyenne $27,1 \%$ pour les premières et $73 \%$ pour les seconds. Les taxons inventoriées et identifiés sont à un taux de $58 \%$, ce qui donne une image non négligeable de la végétation locale quoique inférieur au taux de $80 \%$ préconisé par Reille et Pons (1990). De plus, les taxons marqueurs de la forêt inondable comme : Pycnanthus angolense (4\%), Macaranga heudolotii (2,8\%), Chrysobalanus icaco var. ellipticus $(2,1 \%)$, Parinari robusta $(1,8 \%)$ sont bien présents mais à des taux relativement faibles. Alchornea cordifolia, à un taux de $25,5 \%$, est l'espèce dominante du spectre pollinique de cette formation végétale. Elle est considérée comme typiquement pionnière et héliophile par Vincens et al. (2000). Les taxons allochtones se retrouvent à 58,3\%. Parmi ceux-ci, on retrouve des taxons de mangrove (Rhizophora racemosa et Acrostichum aureum), de fourrés (Bridelia ferruginea, Chaetachme aristata, Sabicea africana, etc.), 
de plantation (Elaeis guineensis, Senna siamea, Chrysophyllum albidum, Pinus caribaea, etc.), et de forêt (Ceiba pentandra, Celtis mildbraedii, etc.). Ces différentes formations font partir de la végétation environnante.

\section{Mangrove}

\section{Inventaire floristique}

Deux sites sont prospectés: l'un à Djègbadji dans la mangrove de la lagune côtière et l'autre à Agatogbo dans une mangrove très dégradée au bord du lac Ahémé. Au total, 49 espèces sont inventoriées (Tableau 2). Les espèces les plus fréquentes sont: Rhizophora racemosa, Avicennia africana, Machaerium lunatum, Acrostichum aureum, Cyperus articulatus, Paspalum vaginatum. En lisière, sur terre ferme, on recense: Ipomoea asarifolia, Spermacoce verticillata, Tephrosia purpurea, Capraria biflora, Elaeis guineensis, etc.

\section{Spectre pollinique}

A l'analyse pollinique des 6 échantillons, 43 taxons sont identifiés (Tableau 2). Les pourcentages de taxons d'herbacées, d'arbres et arbustes sont variables. Ils se situent entre $45,5 \%$ et $72,7 \%$ pour les herbacées et $27,3 \%$ et $54,6 \%$ pour les arbres et arbustes, soit en moyenne $56,3 \%$ pour les premiers et $43,8 \%$ pour les seconds. Le fort taux d'herbacées est dû à l'état de dégradation de la mangrove béninoise dont les zones déboisées sont colonisées par la prairie marécageuse. Les taxons les plus abondants $\mathrm{du}$ spectre pollinique sont par ordre d'importance : Spermacocce verticillata (18,4\%), Hyptis suaveolens (16,6\%), Paspalum vaginatum (8,1\%), Acrostichum aureum (7\%), Ipomoea asarifolia (5,9\%), Cyperaceae (5,6\%), Elaeis guineensis (4,3\%), Polygala arenaria (1,4\%), Cocos nucifera $(1,3 \%)$, Sida rhombifolia $(1,1 \%)$, etc. En considérant l'inventaire floristique et l'analyse pollinique, on note que la représentativité locale du dépôt pollinique de la formation n'est que de $42,9 \%$ contre $51,2 \%$ d'espèces seulement identifiées, c'est-à-dire relevant d'un apport extra-local au sens de Heim (1970). Au nombre de ces taxons allochtones, on peut citer: Symphonia globulifera, Parinari robusta, Mitragyna inermis, Raphia hookeri relevant de la forêt marécageuse, Chrysobalanus icaco var. orbicularis, Polygala arenaria, Chenopodium ambrosioides provenant de fourré secondaire, Cola gigantea de forêt dense humide semidécidue et Cocos nucifera, Elaeis guinensis, issus des plantations. Les taxons caractéristiques de mangrove que sont: Rhizophora racemosa (0,8\%), Avicennia africana $(0,1 \%)$ sont très faiblement représentés. Ceci confirme le point de vue de Frédoux (1980) qui stipule que les pollens de Rhizophora peuvent être absents des spectres polliniques des sédiments de surface prélevés en mangrove.

\section{Forêt marécageuse \\ Inventaire floristique}

Trois forêts marécageuses dégradées pour la plupart sont prospectées, l'une à Adjavié, la deuxième à Dogbocomey et la troisième à Goho. La liste des 105 espèces inventoriées est donnée dans le Tableau 6. Les plus abondantes sont: Alchornea cordifolia, Anthocleista vogelii, Sabicea africana, Ficus capreifolia, Bambusa vulgaris, etc. Le site de Goho renferme des espèces différentielles comme Rhizophora racemosa (planté au cours d'un projet de rénovation des berges) et Acrostichum aureum, témoin d'une ancienne mangrove ainsi que des espèces de milieux marécageux telles que Typha australis, Centella asiatica, Lygodium microphyllum et Mimosa pigra. Non loin de cette forêt, sont observés des fourrés secondaires d'où émergent des espèces reliques de forêt dense semi-décidue telles que Ceiba pentandra, Cola gigantea, Cola millenii, Chrysophyllum albidum et Celtis spp. 


\section{Spectre pollinique}

De l'analyse pollinique des quatre échantillons, 67 taxons sont identifiés (Tableau 2). Les taux de taxons d'herbacées, d'arbres et arbustes se situent entre $47,4 \%$ et $55 \%$ pour les premiers et $45 \%$ et $52,7 \%$ pour les seconds, soit respectivement en moyenne $51,8 \%$ et $48,2 \%$. Le taux élevé des herbacées indique un état dégradé de la forêt marécageuse. Les taxons les plus abondants $\mathrm{du}$ spectre pollinique sont par ordre d'importance : Elaeis guineensis (41\%), Poaceae $(25,6 \%)$ et les Spores $(23,4 \%)$. Les taxons inventoriées et identifiés font $24,8 \%$, ce qui dénote une faible représentativité de la végétation locale. Les taxons allochtones, à $59,7 \%$, mettent plus en exergue la végétation extra-locale ou régionale. Ils sont regroupés en taxons de forêt dense humide semi-décidue (Cola gigantea, Triplochiton scleroxylon et Pycnanthus angolense,), de fourrés (Macrosphyra longistyla, Flabellaria paniculata, Sabicea africana, etc.), de plantation (Elaeis guineensis, Cocos nucifera) et de prairie marécageuse (Ludwigia stolonifera, Nymphaea lotus, Hygrophila auriculata, etc.). Les principaux taxons caractéristiques de forêt marécageuse tels que : Alstonia congensis $(0,1 \%)$, Anthocleista vogelii $(0,02 \%)$, Symphonia globulifera $(0,9 \%)$ et Carapa procera sont bien présents mais à de très faibles taux.

\section{Prairie marécageuse Inventaire floristique}

La prairie marécageuse est issue de la dégradation de la forêt marécageuse ; elle est rencontrée à Kraké. L'inventaire floristique a permis de recenser 22 espèces (Tableau 2). Les espèces les plus fréquentes sont : Cyclosorus striatus, Lasiomorpha senegalensis, Dissotis rotundifolia, Centella asiatica, Ipomoea aquatica, Lygodium microphyllum, Clappertonia ficifolia, auxquelles s'entremêlent par endroits Anthocleista vogelii et Elaeis guineensis.

\section{Spectre pollinique}

Au total, 61 taxons sont identifiés dans les deux échantillons (Tableau 2). Le taux de taxons d'herbacées se situe entre $37,3 \%$ et $44,4 \%$ et celui d'arbres et arbustes entre $55,6 \%$ et $62,8 \%$, soit en moyenne respectivement $40,8 \%$ et $59,2 \%$. Les taxons les plus fréquents du spectre pollinique sont par ordre d'importance: Poaceae (51\%), Spores (20,2\%), Elaeis guineensis (17,2\%), Alchornea cordifolia $(1,1 \%)$, etc. Les taxons inventoriées et identifiés sont à un taux de 40,9\% ; le dépôt pollinique ne donne qu'une image moyenne de la formation. Les taxons seulement identifiés font un taux de $85,3 \%$; ce qui indique un apport extérieur (extra-local, régional ou lointain) très important. Parmi ces taxons allochtones, on retrouve des espèces de forêt dense humide semi-décidue (Funtumia elastica et Triplochiton scleroxylon), de forêt marécageuse (Alstonia congensis, Raphia hookeri, Symphonia globulifera, Vitex grandifolia, etc.), de plantation (Acacia auriculiformis, Cocos nucifera) et des rudérales (Crotalaria retusa, Hyptis suaveolens, Oldenladia herbacea, Cleome gynandra, Sida linifolia, Scoparia dulcis, etc.). La présence de pollens d'espèces de forêt marécageuse dans la prairie peut s'expliquer, soit par leur dépôt par des arbres qui les auraient produits et qui auraient été coupés, soit par un apport lointain par le vent ou l'eau. Les taxons marqueurs de la prairie marécageuse comme Clappertonia ficifolia, Lygodium microphyllum, Sabicea calycina, Spermacocce verticillata, Tetracera alnifolia, Typha australis et Alchornea cordifolia sont représentés mais à des taux faibles.

\section{Affinités entre les échantillons}

Le dendrogramme issu de la classification hiérarchique montre cinq groupes d'échantillons de surface sur la base de leur ressemblance pollinique (Figure 3) qui sont : 
- groupe 1: composé de 5 échantillons dont $60 \%$ sont issus de plantation (Ah1, Ah2 et Ah3), $20 \%$ de forêt dense humide semidécidue (Ad3) et $20 \%$ d'échantillons de forêt inondable (Ah6) ; il est à dominance d'échantillons de plantation;

- groupe 2: composé de 7 échantillons dont 85,7\% d'échantillons de forêt inondable (Ah8, Ah9, Ah10, Ah11, Ah12, Ah13) et 14,3\% d'échantillons de forêt dense humide semidécidue (Ni3) ; il est à dominance d'échantillons de forêt inondable ;

- groupe 3: composé de 8 échantillons, subdivisés en deux sous-groupes; globalement $37,5 \%$ des échantillons relèvent de la mangrove (Ag1, Ou2, Ou3), 37,5\% de la forêt denses humide semi-décidue (Ad4, Ad6, Zi1) et $25 \%$ de forêt inondable (Ah5, Ah7) ; c'est un groupe mixte ;

- groupe 4 : composé de 6 échantillons (Ad1, Ad2, Ad5, Ad7, Ni1, Ni2) tous de la forêt dense humide semi-décidue. ; il relève de la forêt dense humide semi-décidue ;

- groupe 5: comprend 7 échantillons subdivisés aussi en deux sous-groupes, mais globalement 4 échantillons (soit 57,14) sont prélevés sur d'anciens sites de mangrove ou de mangrove très dégradée (Ou1, Ou4, Go1 et $\mathrm{Ag} 2$ ) et 3 échantillons (soit 42,9\%) sont issus de prairie marécageuse $(\mathrm{Kr} 1, \mathrm{Kr} 2)$ et de forêt marécageuse très dégradée (Do1), ce groupe est à dominance de relevés de prairie.

Tableau 2 : Liste floristique.

\begin{tabular}{|c|c|c|c|c|c|c|c|c|c|c|c|}
\hline \multirow[t]{2}{*}{ Espèces } & \multicolumn{2}{|c|}{ FDHSD } & \multicolumn{2}{|c|}{$\mathbf{P I}$} & \multicolumn{2}{|c|}{ FI } & \multicolumn{2}{|c|}{ Ma } & \multicolumn{2}{|c|}{ FM } & $\mathbf{P M}$ \\
\hline & $\mathbf{F r}$ & Inv & $\mathbf{F r}$ & Inv & $\mathbf{F r}$ & Inv & $\mathbf{F r}$ & Inv & Fr & Inv & $\begin{array}{ll}\text { Fr } & \text { Inv }\end{array}$ \\
\hline \multicolumn{12}{|l|}{ Acanthaceae } \\
\hline Asystasia gangetica (L.) T. & & & & & & & & & 006 & $\mathrm{x}$ & \\
\hline Anderson & & & & $\mathrm{x}$ & & $\mathrm{x}$ & & & & & \\
\hline Hygrophila auriculata & & & & & & & & & 0,02 & & \\
\hline \multicolumn{12}{|l|}{ (Schumach.) Heine } \\
\hline Justicia insularis T. Anderson & 0,1 & & 0,12 & & 0,03 & $\mathrm{x}$ & & & & & \\
\hline \multicolumn{12}{|l|}{ Agavaceae } \\
\hline Dracaena arborea Bak. & 0,1 & & & & 0,1 & & & & & & \\
\hline \multicolumn{12}{|l|}{ Amaranthaceae/Chenopodiaceae } \\
\hline Amaranthaceae/Chenopodiaceae- & 5,65 & & & & & & 0,33 & & 0,15 & & \\
\hline type & & & 1,33 & & 0,64 & & & & & & \\
\hline \multicolumn{12}{|l|}{ Amaryllidaceae } \\
\hline Crinum jagus (J. Thomps.) Dandy & & & & & & & & & 0,02 & $\mathrm{x}$ & 0,08 \\
\hline \multicolumn{12}{|l|}{ Anacardiaceae } \\
\hline Lannea nigritana (Scott-Elliot) & & $\mathrm{x}$ & & & 0,05 & $\mathrm{x}$ & & & & $\mathrm{x}$ & \\
\hline \multicolumn{12}{|l|}{ Keay } \\
\hline Mangifera indica $\mathrm{L}$. & & & & & 0,03 & & & & & $\mathrm{x}$ & 0,03 \\
\hline Sorindeia grandifolia Engl. & & $\mathrm{x}$ & & $\mathrm{x}$ & & & & & & $\mathrm{x}$ & \\
\hline Spondias mombin $\mathrm{L}$. & 0,1 & & & & & & & & 0,02 & & \\
\hline \multicolumn{12}{|l|}{ Annonaceae } \\
\hline Annona senegalensis Pers. & & & & & & $\mathrm{x}$ & & $\mathrm{x}$ & & $\mathrm{x}$ & \\
\hline Artabotrys velutinus Scott-Elliot & & $\mathrm{x}$ & & & & $\mathrm{x}$ & & & & & \\
\hline
\end{tabular}


Cleistopholis patens (Benth.)

Engl. \& Diels

Monanthotaxis parviflora (Oliv.)

Verdc.

Monodora myristica (Gaertn.)

Dunal

Monodora tenuifolia Benth

Uvaria chamae P. Beauv.

Xylopia aethiopica (Dunal) A.

Rich.

\section{Apiaceae}

Centella asiatica (L.) Urb.

\section{Apocynaceae}

Alstonia congensis Engl.

Baissea axillaris (Benth.) Hua

Carissa spinarum L.

Funtumia elastica (Preuss) Stapf

Hollarhena floribunda (G. Don)

Durand \& Schinz

Landolphia dulcis (Sabine) Pichon

Motandra guineensis (Tonn.) A.

DC.

Rauvolfia vomitoria Afzel.

Strophanthus sarmentosus DC.

Voacanga africana Stapf

\section{Araceae}

Anchomanes difformis (Blume)

Engl.

Cercestilis mirabilis (N.E.Br.)

Bogner

Culcasia saxatilis A. Chev.

Lasiomorpha senegalensis Schott

Araceae-type

\section{Arecaceae}

Arecaceae-type

Borassus aethiopum Mart.

Calamus deerratus G. Mann \& H.

Wendl.

Caryota urens L.

Cocos nucifera $\mathrm{L}$.

Elaeis guineensis Jacq.

Eremospatha macrocarpa (G.

Mann \& H. Wendl.) H. Wendl. $\mathrm{x}$

$\mathrm{X}$

$\mathrm{x}$

$\begin{array}{lll}\mathrm{x} & 0,2 \quad \mathrm{x}\end{array}$
$\mathrm{X}$

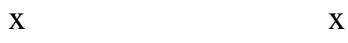

X $\quad$ X $0,03 \quad x$

X

\section{$0,04 \quad \mathrm{x} \quad 0,21$}

$\mathrm{X}$

$\mathrm{x}$

$\mathrm{x}$

$0,1 \quad \mathrm{x}$

$0,08 \quad x$

$\mathrm{X}$

X $\quad 0,05$

$0,48 \quad 0,38$

$\mathrm{X}$

$\mathrm{x}$

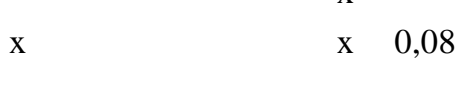

$\mathrm{X}$ 
Laccosperma secundiflorum (P.

Beauv.) Kuntze

Phoenix reclinata Jacq.

$\mathrm{x}$

Raphia hookeri G. Mann \& H.

Wendl.

\section{Asclepiadaceae}

Pergularia daemia Afzel.

Periploca nigrescens (Forssk.)

$\mathrm{X}$

Chiov.

$\mathrm{x}$

Secamone afzelii (Schult.) K.

$x$
$\mathrm{x}$

Schum.

$0,12 \quad x$

\section{Asteraceae}

Acanthospermum hispidum DC.

Aspilia rudis Oliv.

Chromolaena odorata (L.) R. M.

King

Compositae

0,12

X

$\mathrm{X}$
0,13

Emilia praetermissa Milne-Redh.

Avicenniaceae

Avicennia germinans (L.) L.

$0,14 \quad x$

\section{Bignoniaceae}

Newbouldia laevis (P. Beauv.)

Seemann ex Bureau

0,08

0,05

Spathodea campanulata P. Beauv.

Bombacaceae

Ceiba pentandra (L.) Gaertn.

$\mathrm{X}$

0,08

x $\quad 0,04 \quad x$

Erhetia cymosa Thonn. ex Schum.

$\mathrm{X} \quad \mathrm{X}$

\section{Campanulaceae}

Campanulaceae-type

$\mathrm{X}$

\section{Capparidaceae}

Cleome gynandra L.

Ritchiea capparoides (Andr.)

Britten

$\mathrm{X}$

Casuarinaceae

Casuarina equisetifolia $\mathrm{L}$.

\section{Cecropiaceae}

Musanga cecropioides Tedlie

$\mathrm{X}$

Celastraceae

Gymnosporia buchananii Loes.

Gymnosporia senegalensis (Lam.)

Loes.

Loeseneriella africana (Willd.) $\mathrm{N}$.

$\mathrm{X}$

hallé 
Salacia pallescens Oliv. $\mathrm{x}$ $\mathrm{X}$

\begin{tabular}{lcccc}
\hline Celtidaceae & & & & \\
Celtis milbraedii Engl. & 0,2 & $\mathrm{x}$ & & \\
Chaetachme aristata Planch. & & & & 0,31 \\
Trema orientalis $($ L. $)$ Blume & & x & x & 0,18
\end{tabular}

\section{Chenopodiaceae}

Chenopodium ambrosioides L.

$\mathrm{X}$

Chrysobalanaceae

Chrysobalanus icaco $\mathrm{L}$.

$2,12 \quad x \quad 0,19 \quad x$

0,61

Maranthes robusta (Oliv.) Prance

$1,76 \times 0,14 \quad 0,04$

ex F. White

\section{Clusiaceae}

Symphonia globulifera L.f.

$\begin{array}{lllll}0,59 & \mathrm{x} & 0,05 & 0,89 & 0,08\end{array}$

\section{Combretaceae}

Combretum racemosum $\mathrm{P}$. Beauv.

Combretum indicum (L.)

DeFilipps

0,12

Combretaceae/Melastomataceae- $\quad 0,1 \quad \mathrm{x}$

0,38

0,24

$0,06 \times 4,05$

type

\section{Commelinaceae}

Commelina benghalensis $\mathrm{L}$.

Commelina diffusa Burm.f.

Commelina erecta $\mathrm{L}$.

Palisota hirsuta (Thunb.) K.

Schum.

\section{Connaraceae}

Agelaea pentagyna (Lam.) Baill.

Cnestis ferruginea Vahl ex DC.

$\begin{array}{rrrrr} & \mathrm{x} & \mathrm{x} & 0,18 & \mathrm{x} \\ 0,1 \quad \mathrm{x} & \mathrm{x} & \mathrm{x} \\ & & & & \\ & & & \end{array}$

Schumach.) Benth.

$\mathrm{x}$

\section{Convolvulaceae}

Calycobolus africanus (G. Don)

heine

Hewetia scandens (Milne)

Mabberley

Ipomoea aquatica Forssk.

Ipomoea asarifolia (Desr.) Roem.

$\&$ Schult.

Ipomoea fistulosa Mart. ex Choisy

Xenostegia tridentata (L.) Austin

\& Staples

\section{Cucurbitaceae}

Luffa cylindrica (L.) M. Roem. 


\begin{tabular}{|c|c|c|c|c|c|c|c|c|c|c|c|c|}
\hline Cyperaceae & & & & & & & & & & & & \\
\hline Cyperus distans L.f. & & & & $\mathrm{x}$ & & & & & & & & \\
\hline Cyperus ligularis L. & & & & & & & & $\mathrm{x}$ & & & & \\
\hline Fuirena umbellata Rottb. & & & & & & & & & & $\mathrm{x}$ & & \\
\hline Kyllinga erecta Schumach. & & & & & & & & $\mathrm{x}$ & & $\mathrm{x}$ & & \\
\hline Rynhchospora corymbosa (L.) & & & & & & & & & & $\mathrm{x}$ & & \\
\hline Britt. & & & & & & & & & & & & \\
\hline Scleria sp. & & & & & & & & & & $\mathrm{x}$ & & \\
\hline Cyperaceae-type & 9,9 & & 7,86 & & 2,73 & $\mathrm{x}$ & 5,55 & $\mathrm{x}$ & 0,89 & $\mathrm{x}$ & 0,66 & \\
\hline Dichapetalaceae & & & & & & & & & & & & \\
\hline $\begin{array}{l}\text { Dichapetalum madagascariense } \\
\text { Poir. }\end{array}$ & & $\mathrm{x}$ & & & & & & & & & & \\
\hline Dilleniaceae & & & & & & & & & & & & \\
\hline Tetracera alnifolia Willd. & 0,1 & & & & 0,05 & & & & 0,08 & $\mathrm{x}$ & 0,08 & $\mathrm{x}$ \\
\hline Ebenaceae & & & & & & & & & & & & \\
\hline Diospyros tricolor (Schum. \& & & & & & 1,05 & $\mathrm{x}$ & & & & & & \\
\hline Thonn.) Hiern & & & 0,12 & $\mathrm{x}$ & & & & & & & & \\
\hline Euphorbiaceae & & & & & & & & & & & & \\
\hline $\begin{array}{l}\text { Alchornea cordifolia (Schumach. } \\
\& \text { Thonn.) Müll. Arg. }\end{array}$ & 0,71 & & 1,45 & $\mathrm{x}$ & 25,5 & $\mathrm{x}$ & 0,19 & & 0,96 & $\mathrm{x}$ & 1,12 & $\mathrm{x}$ \\
\hline $\begin{array}{l}\text { Aleurites moluccana (L.) Willd. } \\
\text { Antidesma membranaceum Müll. }\end{array}$ & & $\mathrm{x}$ & & & & & & & & & & \\
\hline Arg. & & & 1,45 & $\mathrm{x}$ & & & & & & & & \\
\hline Bridelia ferruginea Benth. & & $\mathrm{x}$ & 0,12 & & 0,23 & & & & & & & \\
\hline Croton lobatus $\mathrm{L}$. & & & & & & & & & 0,02 & & & \\
\hline Drypetes floribunda (Müll. Arg.) & 0,1 & & & & 0,05 & & & & & & & \\
\hline Hutch. & & & & & & & & & & & & \\
\hline Flueggea virosa (Roxb. ex Willd.) & & $\mathrm{x}$ & 0,24 & & 0,03 & & & & & $\mathrm{x}$ & & \\
\hline $\begin{array}{l}\text { Hevea brasilensis (Willd. ex A. } \\
\text { Juss.) Müll. Arg. }\end{array}$ & & $\mathrm{x}$ & & & & & & & & & & \\
\hline Jatropha gossypiifolia $\mathrm{L}$. & & & 0,12 & & & & 0,05 & $\mathrm{x}$ & 0,49 & & & \\
\hline Macaranga heudelotii Baill. & & & 0,12 & $\mathrm{x}$ & 2,78 & $\mathrm{x}$ & & & & & & \\
\hline Mallotus oppositifolius (Geisel.) & 1,72 & $\mathrm{x}$ & & & & & 0,05 & & & & 0,03 & \\
\hline Müll. Arg. & & & & $\mathrm{x}$ & & & & & & & & \\
\hline Margaritaria discoidea (Baill.) & & & & & & & & & & $\mathrm{x}$ & & \\
\hline Webster & & & & & & & & & & & & \\
\hline Phyllanthus mиellerianus (Kuntze) & 0,1 & & & & & & & $\mathrm{x}$ & & & & \\
\hline Exell & & & & & & & & & & & & \\
\hline Uapaca togoensis $\mathrm{Pax}$ & & & & & & & & & & & 0,16 & \\
\hline Icacinaceae & & & & & & & & & & & & \\
\hline $\begin{array}{l}\text { Raphiostylis beninensis (Hook.f. } \\
\text { ex Planch.) Planch. ex Benth. }\end{array}$ & & & & $\mathrm{x}$ & & $\mathrm{x}$ & & & & $\mathrm{x}$ & & \\
\hline
\end{tabular}




\section{Irvingiaceae}

Irvingia gabonensis (Aubry-

0,1

$\mathrm{X}$

Lecomte ex O'Rorke) Baill.

\section{Lamiaceae}

Hyptis lanceolata Poir. $2,27 \quad x$

Hyptis suaveolens (L.) Poit.

$0,2 \quad 0,12$

$0,1 \quad 16,6 \quad x$

0,13

\section{Lecythidaceae}

Napoleonaea lvogelii Hook. \&

Planch.

\section{Leg.-Caesalpinioideae}

Bauhinia-type

0,03

Caesalpiniaceae

$0,4 \quad \mathrm{x}$

Cassia sieberiana DC.

Chamaecrista mimosoides (L.)

Greene

Chamaecrista rotundifolia (Pers.)

Greene

Delonix regia (Boj. ex Hook.) raf.

Dialium guinense Willd.

Senna siamea (Lam.) H. S. Irwin \& Barneby

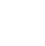

Leg.-Mimosoideae

Acacia auriculiformis A. Cunn. ex $\quad 0,3$

Benth.

Albizia adianthifolia (Schumach.)

W. F. Wright

Albizia coriaria Oliv.

$0,12 x$

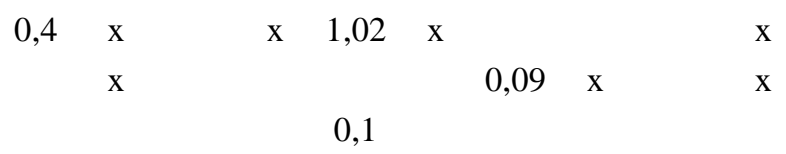

Albizia ferruginea (Guill. \& Perr.)

Benth.

Albizia glaberrima (Schumach. \&

Thonn.) Benth.

X $\quad$ X 0,03

Albizia zygia (DC.) J. F. Macbr.

Albizia-type

Entada abyssinica Steud. ex A.

$\begin{array}{rrrr}9,79 & \mathrm{X} & 2,48 & \mathrm{x} \\ & & & \\ & & 0,1 & \mathrm{X} \\ & & \mathrm{X}\end{array}$

$\mathrm{X}$

Rich.

Mimosa pigra $\mathrm{L}$.

Parkia bicolor A. Chev. X

Pentaclethra macrophylla Benth. $\quad 1,41 \quad \mathrm{x}$

Schrankia leptocarpa DC.

Tetrapleura tetraptera (Schumach. 0,3 \& Thonn.) Taub.

\section{Leg.-Papilionoideae}

Abrus precatorius L. 
Angylocalyx oligophyllus (Baker)

Baker.f.

Calopogonium mucunoides DC.

Crotalaria retusa $\mathrm{L}$.

Dalbergia ecastaphyllum (L.)

Taub.

Dalbergia saxatilis Hook.f.

Dalbergia setifera Hutch. \&

Dalziel

Desmodium ramosissimum G. Don $\quad 0,1$

Desmodium-type

Dolichos trilobus L.

Indigofera hirsuta L.

Indigofera tinctoria $\mathrm{L}$.

Lonchocarpus sericeus (Poir.)

Kuth

Machaerium lunatum (L.f.) ucke Millettia thonningii (Schumach. \&

Thonn.) Baker

Mucuna pruriens (L.) DC.

Neorautanenia mitis (A. Rich.)

Verdc.

Pterocarpus santalinoides L'Hér.

ex DC.

Sesbania leptocarpa DC.

Sesbania-type

Tephrosia purpurea (L.) Pers.

\section{Lentibulariaceae}

Utricularia reflexa Oliv.

\section{Loganiaceae}

Anthocleista vogelii Planch.

Strychnos floribunda Gilg

0,1

Usteria guineensisWilld.

\section{Loranthaceae}

Tapinanthus globiferus (A. Rich.)

0,1

$0,12 x$

\section{1}

Tiegh.

0,08

Lycopodiaceae

Lycopodiella cernua (L.) Pic.

$\mathrm{X}$

Serm.

\section{Malpighiaceae}

Flabellaria paniculata Cav.

0,51

0,64

0,04

\section{Malvaceae}

Hibiscus surattensis L. 
Sida acuta Burm.f. 0,51

Sida linifolia Juss. ex Cav.

$0,12 \quad 0,33$

$0,06 \quad \mathrm{x}$

$\mathrm{X}$

Sida rhombifolia $\mathrm{L}$.

$1,14 \quad \mathrm{x}$

Malvaceae-type

Marantaceae

Hypselodelphys violacea (Ridl.)

Milne-Redh.

\section{Melastomataceae}

Heterotis rotundifolia (Sm.) Jacq.-

Fél.

Melastomastrum segregatum

(Benth.) A. \& R. Fern.

\section{Meliaceae}

Carapa procera DC.

Entandophragma angolense

(Welw.) C. DC.

Trichilia megalantha Harms

$\mathrm{X}$

Trichilia prieureana A. Juss

$\mathrm{X}$

$0,15 \quad x$

\section{Menispermaceae}

Sphenocentrum jollyanum Pierre

\section{$\mathrm{X}$}

Trichilia subcordata Oliv.

\section{Moraceae}

Antiaris toxicaria Lesch.

Artocarpus altilis (Parkinson)

Fosberg

Ficus capreifolia Delile

Ficus exasperata Vahl

Ficus sur Forssk.

Milicia excelsa (Welw.) C. C.

Berg

Trilepisium madagascariensis DC.

\section{Moringaceae \\ Moringa oleifera Lam.}

Myristicaceae

Pycnanthus angolensis (Welw.) $\quad 0,4 \quad \mathrm{x}$

$0,4 \quad x$

Warb.

Myrtaceae

Psidium guajava $\mathrm{L}$.

Syzygium guineense (Willd.) DC. $\quad 0,1$

var. littorale (Aubrév.) Keay

\section{Nephrolepidaceae}

Nephrolepis biserrata (Sw.)

Schott
0,05

$\mathrm{X}$

X

X

$\mathrm{X}$

$\mathrm{X}$
$\mathrm{X}$

\section{X}




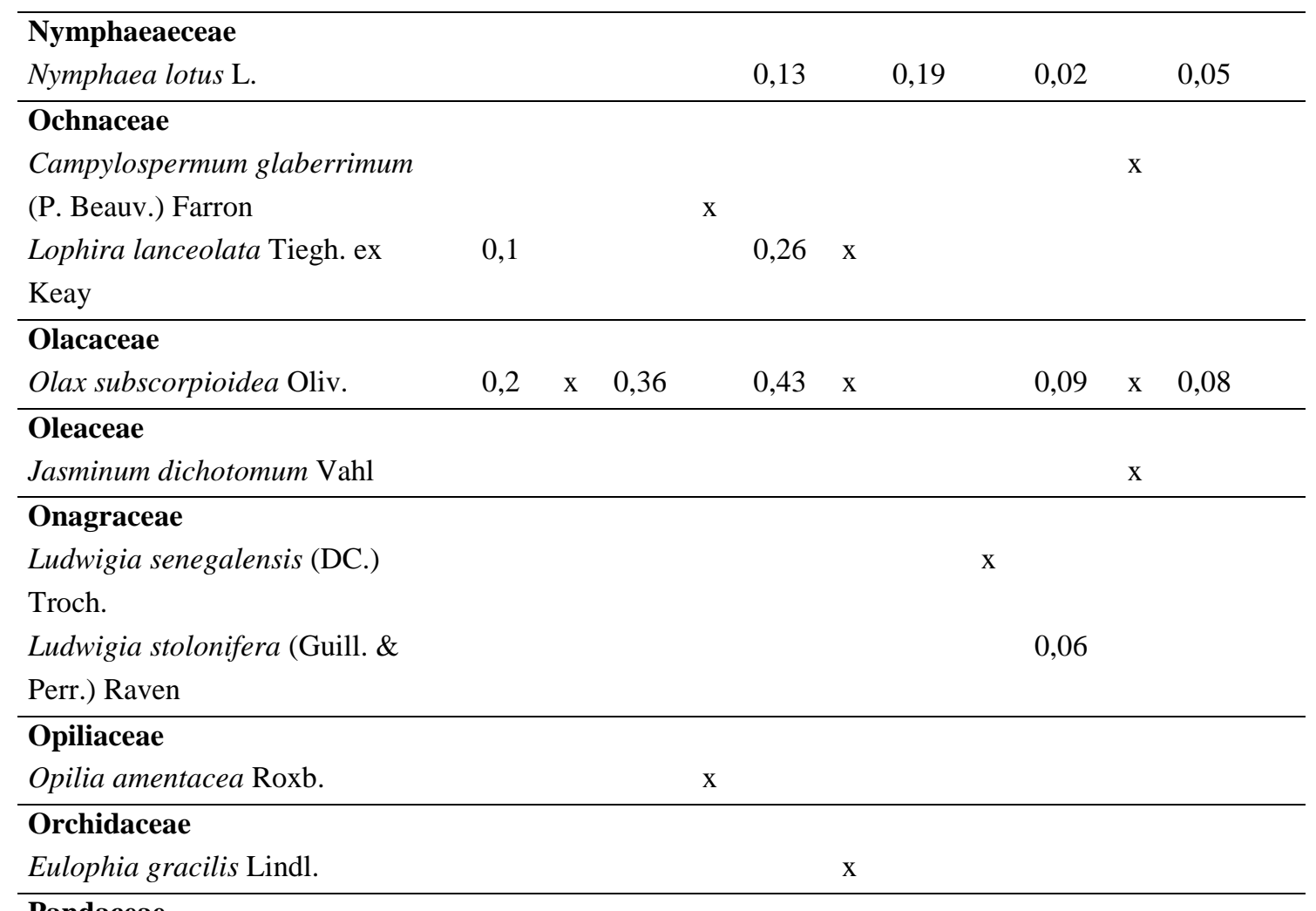

\section{Pandaceae}

Microdesmis keayana J. Léonard

$\mathrm{X}$

Passifloraceae

Adenia lobata (Jacq.) Engl.

$\begin{array}{llllll}0,81 & \times & 43,3 & & 6,07 & \\ & & 0,36 & \times & 0,94 & \text { X } \\ & & 0,24 & & & \end{array}$

Barteria nigritana Hook.f.

0,24

\section{Pedaliaceae}

Pedalium murex D. Royen ex L.

\section{Pinaceae}

Pinus caribaea Morelet

0,12

0,03

\section{Piperaceae}

Piper guineense Schumach. \&

Thonn.

\section{Poaceae}

Andropogon gayanus Kunth

Bambusa vulgaris Schrad. ex

Wendel

Eragrostis tremula (Lam.) Steud.

Imperata cylindrica (L.) P. Beauv.

Paspalum vaginatum $\mathrm{Sw}$.

$\mathrm{X}$

Saccharum officinarum L.

Schizachyrium sanguineum (Retz.)

Alston 
Sporobolus pyramidalis P. Beauv.

Vitiveria nigritana (Benth.)

Zea mays L.

Poaceae-type

Polygalaceae

Carpolobia lutea G. Don

Polygala arenaria Willd.

Polygonaceae

Persicaria senegalensis (Meisn.)

0,05

Sojak

\section{Polypodiaceae}

Microsorum scolopendria

$\mathrm{X}$

(Burm.f.) Copel

\begin{tabular}{|c|c|c|c|c|c|c|c|c|}
\hline \multicolumn{9}{|l|}{ Portulacaceae } \\
\hline Talinum triangulare (Jacq.) Willd. & 0,4 & 0,24 & & 0,15 & & & & 0,05 \\
\hline \multicolumn{9}{|l|}{ Pteridaceae } \\
\hline Acrostichum aureum $\mathrm{L}$. & 0,71 & 0,36 & $\mathrm{x}$ & 0,2 & 7,02 & $\mathrm{x}$ & 1,19 & $\mathrm{x}$ \\
\hline Ceraptopteris thalictroides (L.) & 1,82 & & & & & & 0,21 & \\
\hline Brongn. & & 0,6 & & & & & & \\
\hline
\end{tabular}

\section{Rhizophoraceae}

Cassipourea barteri (Hook.) N. E.

$\mathrm{Br}$.

Rhizophora racemosa G. Mey.

Rubiaceae

Chassalia kolly (Schumach.)

Hepper

Cremaspora triflora (thonn.) K.

Schum.

Cuviera macroura K. Schum.

Diodia sarmentosa Sw.

Gardenia nitida Hook.

Ixora bractyptera DC.

Keetia hispida (Benth.) Bridson

Macrosphyra longistyla (DC.)

Hiern

Mitracarpus hirtus (L.) DC.

Mitragyna inermis(Willd.) Kuntze

Morelia senegalensis A. Rich. ex

DC.

Morinda lucida Benth.

Oldenladia corymbosa L.

Oldenlandia herbacea (L.) Roxb.

Oxyanthus racemosus (Schumach. $\mathrm{x}$

X

$25,6 \quad x \quad 51$

0,02 


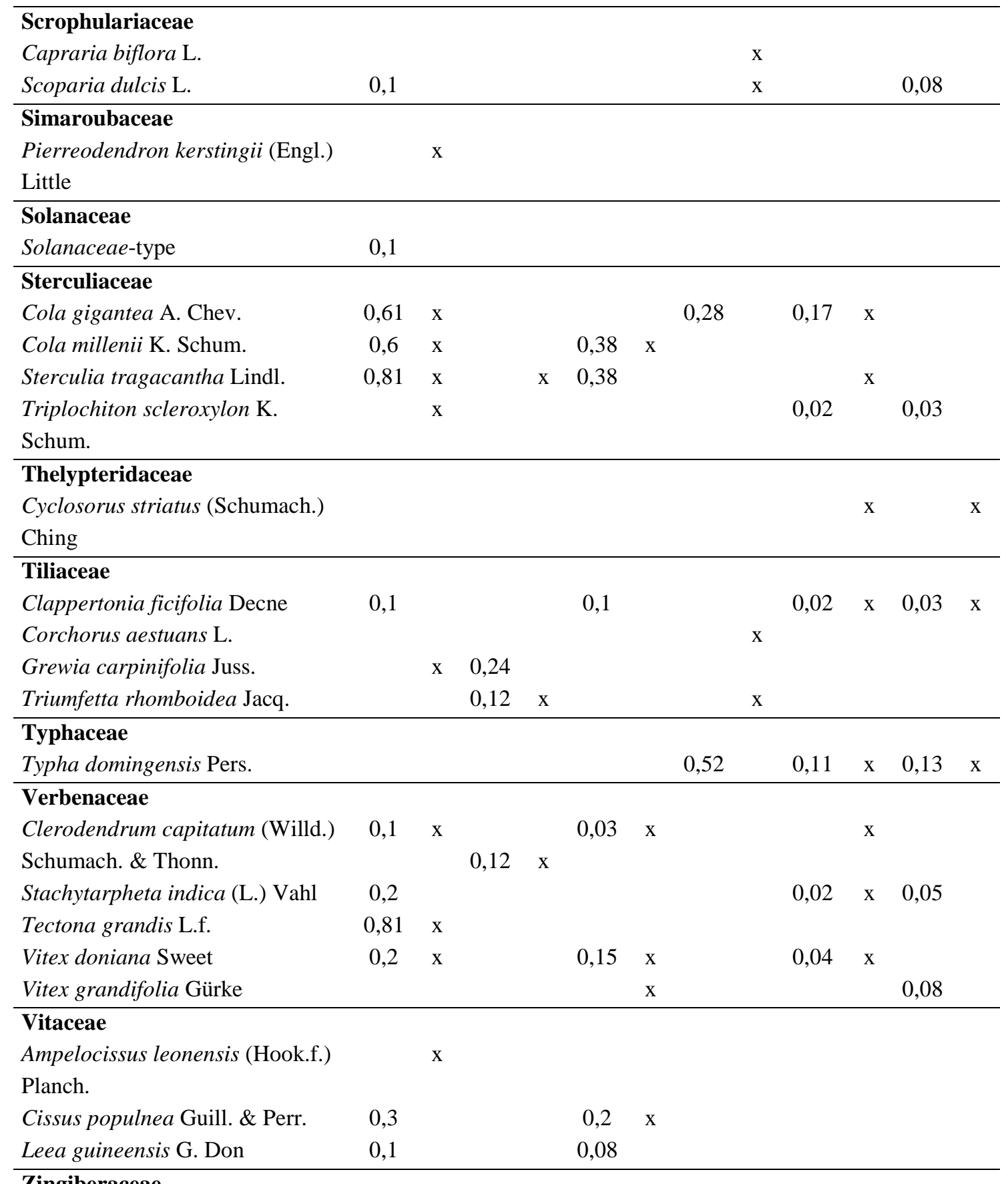

\section{Zingiberaceae}

Aframomum sceptrum (Oliv. \& D.

hanb.) K. Schum.

\begin{tabular}{lccccccc}
\hline Spores & & & & & & & \\
*Spores monolètes & 24 & 0,97 & 5,87 & 0,57 & 19,7 & & 10,7 \\
*Spores trilètes & 2,83 & 0,6 & 1,3 & 0,05 & 3,7 & x & 9,48 \\
\hline
\end{tabular}

FDHSD : Forêt Dense humide Semi-décidue, Pl : plantation, FI : forêt inondable, Ma : mangrove, FM : forêt marécageuse, $\mathrm{PM}$ : prairie marécageuse, Fr : fréquence en pourcentage du taxon, Inv : inventaire floristique (x : présence). 


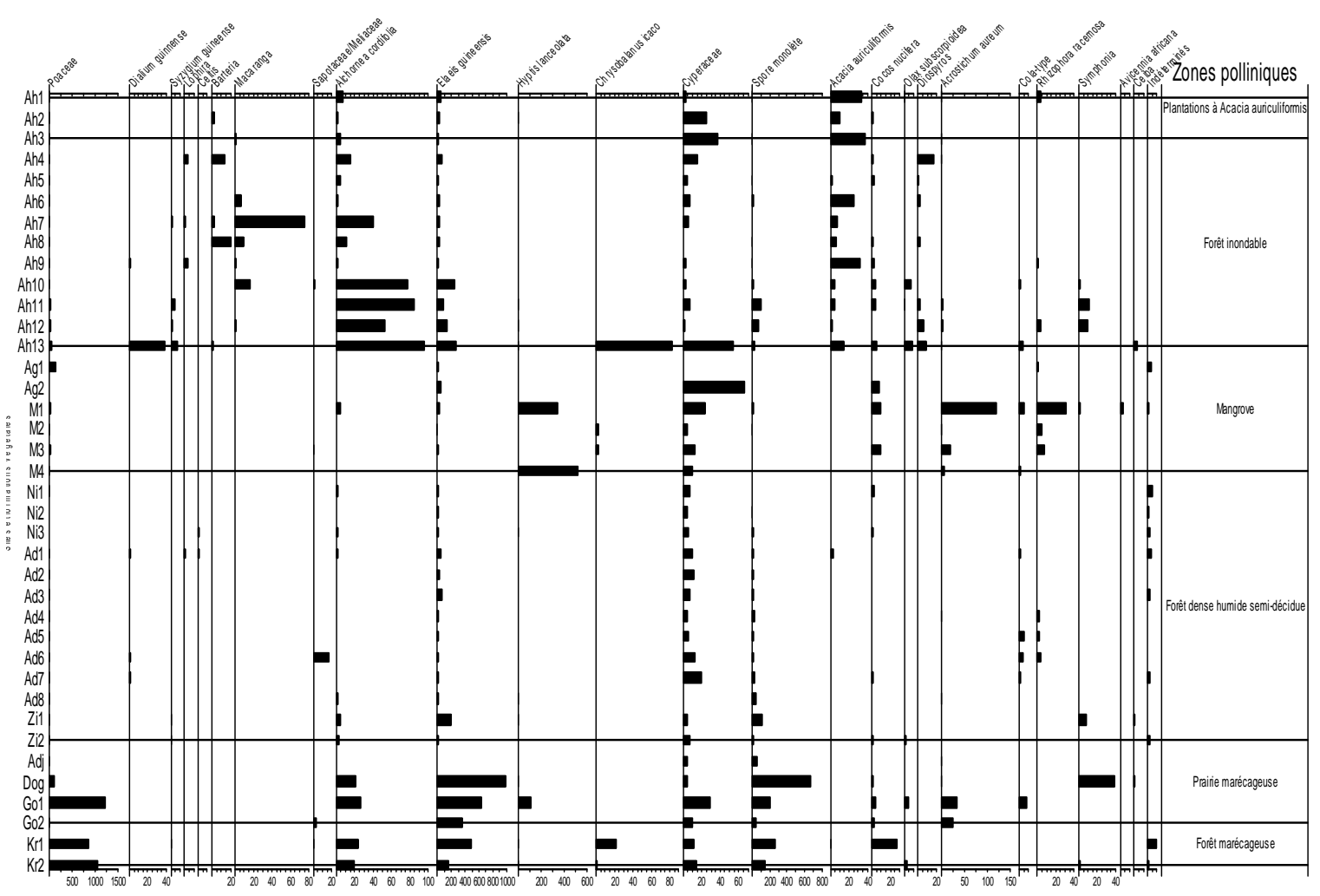

Figure 2 : Diagramme pollinique simplifié des échantillons de surface.

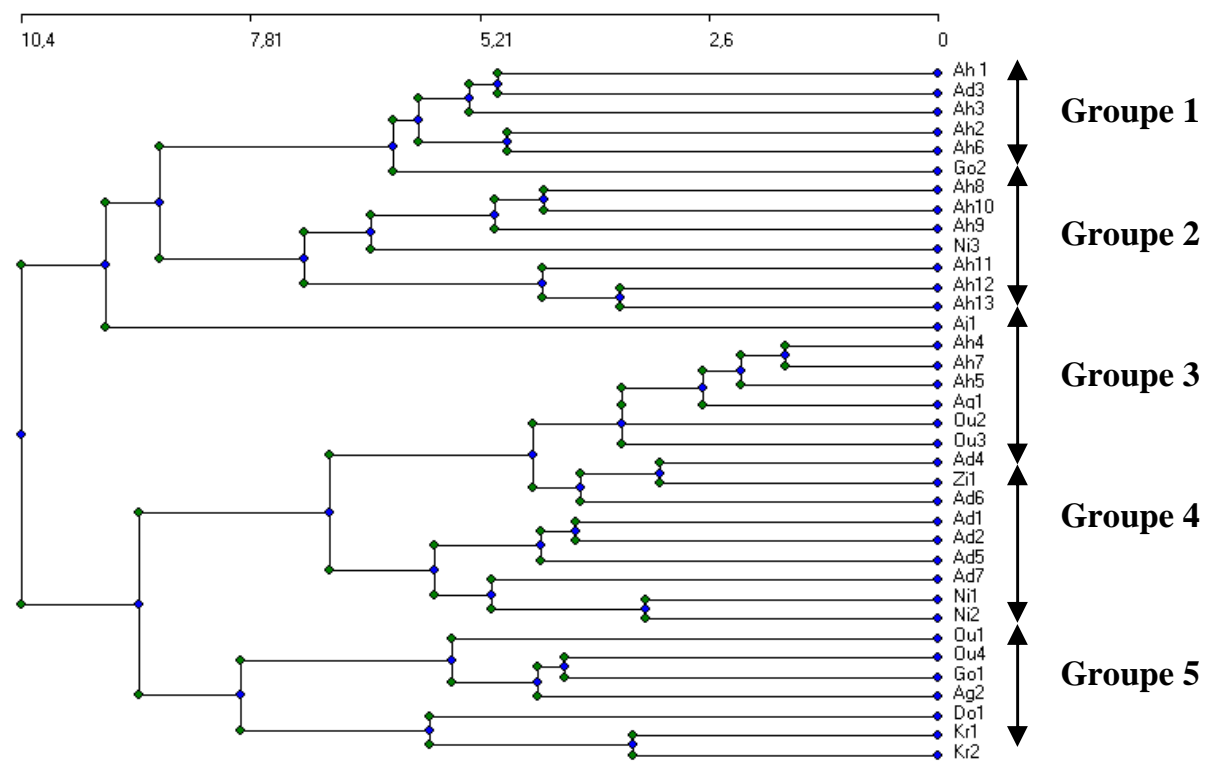

Figure 3 : Dendrogramme des échantillons de surface. 
De l'analyse du dendrogramme, il ressort que le groupe 4, à 100\% d'échantillons de forêt dense humide semi-décidue est le plus homogène mais il n'est constitué que de 6 échantillons sur un total de 13 relevant de la forêt dense humide semi-décidue. Le reste des échantillons s'est réparti comme suit : 1 dans le groupe 1 à dominance de relevés de plantation, 1 dans le groupe 2 à dominance de relevés de forêt inondable, 3 dans le groupe 3 à dominance de relevés de mangrove et de forêt inondable. La forêt se comporte alors comme un écran qui reçoit aussi bien les apports polliniques local, extra-local et régional. Le même phénomène s'observe aussi, mais à des degrés divers, au niveau de la forêt inondable (groupe 2) et de la plantation (groupe 1) qui sont des formations denses fermées.

Le groupe 5 apparaît aussi homogène si l'on considère le milieu hydromorphe dont sont issus tous les échantillons. Les prairies de milieux d'eau douce et d'eau saumâtre semblent avoir un cortège de taxons communs qui sont, soit indicateurs du milieu, donc local, soit du milieu environnant. En effet, les deux sous-groupes distingués sont issus, l'un de prairie marécageuse et de forêt marécageuse très dégradée assimilable à une prairie d'eau douce et le second de mangrove très dégradée assimilable ici à une prairie de milieu d'eau saumâtre.

Le dendrogramme fait transparaître les degrés d'affinité ou de ressemblance pollinique entre les différents relevés et montre bien l'émiettement de la végétation du Sud-Bénin.

\section{Conclusion}

La comparaison des données de la pluie pollinique des différents types de formations végétales a permis de mettre en relation, pluie pollinique et végétation actuelle dans la partie méridionale du Bénin. La forêt inondable a enregistré le plus fort taux de taxons et le plus faible est connu en mangrove. Le plus fort taux de représentativité est retrouvé toujours en forêt inondable alors que le plus faible est en forêt dense humide semi-décidue. La prairie marécageuse a eu le plus fort taux de taxons allochtones $(85,3 \%)$ alors que la mangrove a enregistré le taux le plus faible (51,2\%). Elaeis guineensis est identifié à des taux importants dans tous les types de formations végétales. En effet cette espèce est partout présente dans le paysage actuel du Sud du Bénin.

Le dépôt pollinique actuel obtenu dans cette étude caractérise bien la végétation du Sud du Bénin. Il peut être donc utile à l'interprétation des séquences polliniques holocènes du Dahomey-Gap.

\section{REFERENCES}

Akpagana K. 1989. Recherche sur les forêts denses humides du Togo. Thèse Doctorat, Univ. Bordeaux III, 181p.

Akoègninou A. 2004. Recherches botaniques et écologiques sur les forêts actuelles du Bénin Thèse d'Etat, Univ. de CocodyAbidjan, 326p.

Akoègninou A, Van der Burg WJ, Van Der Masen LJG. 2006. Flore Analytique $d u$ Bénin. Backhuys Publishers, Université d'Abomey-Calavi: Cotonou, Bénin; 1034.

Bonnefille R. 1972. Associations polliniques actuelles et quaternaires en Ethiopie. (Vallées de l'Awash et de l'Omo). Thèse Doct. Es Sci., Univ. Paris VI, 513p.

Caratini C, Guinet P. 1974. Pollens et Spores d'Afrique Tropicale (Trav. Doc.10). CEGET, CNRS: Talence.

Cour P. 1974. Nouvelles techniques de détection des flux et des retombées polliniques de la sédimentation actuelle au Sahara nord-occidental. «Palynology in medecine $»$, Proceed. $3^{\text {rd }}$ Intern. Palyn. Conf., Moscou, 41-58.

Cour P, Duzer D. 1976. Persistance d'un climat hyperaride au Sahara central et méridional au cours de l'Holocène. Rev. Geogr. Phys. Geol. Dyn., 18: 175-198.

Edorh TM. 1986. Végétation et pluie pollinique actuelles au Togo. Thèse d'Université, Aix-Marseille III, 124 p.

Edorh TM, Afidégnon D. 2008. Représentation pollinique de la végétation actuelle des zones humides du sud-est du Togo. Annales des Sciences Agronomiques, 10(1): 51-70.

Elenga H. 1992. Végétation et climat du Congo depuis 24000 ans B.P Analyse 
palynologique de séquences sédimentaires du pays Bateké et du littoral. Thèse Univ. Aix-Marseille III, $238 \mathrm{p}$.

Elenga H, de Namur C, Vincens A, Roux M, Schwartz D. 2000. Use of plots to define pollen-vegetation relationships in densely forested ecosystems of Tropical Africa. Rev. Palaeobot. Palynol., 112: 79-96.

Faegri, Iversen. 1989. Textbook of Pollen Analysis ( $4^{\text {th }}$ edn). Wiley, Chichester: UK ; 328 p.

Frédoux A. 1980. Evolution de la mangrove près d'Abidjan (Côte d'Ivoire) au cours des quarante derniers millénaires. Travaux et documents de Géographie Tropicale. CEGET 39, 49-88.

Grimm EC. 2004. TGView. Springfield (Illinois State Museum).

Heim J. 1970. Les relations entre les spectres polliniques récents et la végétation actuelle en Europe occidentale. Thèse de doctorat, Université de Louvain, $181 \mathrm{p}$.

Jenik J. 1994. The Dahomey-gap : an important issue in african phytogeography. Mem. Soc. Biogeogr. (3ème serie), IV: $125-133$.

Lebamba J, Vincens A, Jolly D, Ngomanda A, Schevin P, Maley J, Bentaled I, Regab M. 2009. Modern pollen rain in savanna and forest ecosystems of Gabon and Cameroon, Central Atlantic Africa. Review of Palaeobotany and Palynology, 153(1-2): 34-45.

Letouzey R. 1968. Etude Phytogéographique $d u$ Cameroun. Lechevalier: Paris.

Lezine A-M. 1981. Le lac Abiyata (Ethiopie). Palynologie et climatologie du Quaternaire récent. Thèse 3è cycle, Univ. Bordeaux I. Géologie du Quaternaire et Préhistoire. 2 vol. (I : 125p., II : 84p.).

Lézine A-M. 1996. La mangrove ouestafricaine, signal des variations du niveau marin et des conditions régionales du climat au cours de la dernière déglaciation. Bull. Soc. Géol. Fr., 167(6): 743-752.

Lézine A-M, Edorh MT. 1991. Modern pollen deposition in West African Sudanian environments. Rev. Palaeobot. Palynol., 67: 41-58.

Maley J. 1972. La sédimentation pollinique actuelle dans la zone du lac Tchad (Afrique Centrale). Pollen et Spores, 14: 263-307.

Rabier J. 1978. Etude sédimentologique de la lagune de Porto-Novo. (République Populaire du (Bénin). Thèse Doct. Univ. de Bordeaux I et Univ. Nat. du Bénin, $140 \mathrm{p}$.

Reille M, Pons A. 1990. Leçons de Palynologie et d'Analyse Pollinique. Ed CNRS: Paris; 206p.

Reynaud-farrera I. 1995. Histoire des paléoenvironnements forestiers du SudCameroun à partir d'analyses palynologiques et statistiques de dépôts holocènes et actuels. Thèse, Univ. Montpellier II, $230 \mathrm{p}$.

Salgado-Labouriau ML. 1979. Modern pollen deposition in the Venezuelan Andes. Grana, 18: 53-58.

Sowunmi MA. 1973. Pollen grains of Nigeria plants I. Woody species. Grana, 13: 145186.

Sowunmi MA. 1995. Pollen grains of Nigeria plants II. Woody species. Grana, 34: 120141.

Tossou MG. 2002. Recherche palynologique sur la végétation holocène du Sud-Bénin (Afrique de l'Ouest). Thèse de l’Université de Lomé, 136 p.

Vincens A, Dubois MA, Guillet B, Achoundong G, Buchet G, Kamgang Kabeyene Beyala V, de Namur C, Riera B. 2000. Pollen-rain-vegetation relationships along a forest-savana transect in southeastern Cameroun, Rev. Palaeobot. Palynol., 110: 191-208.

Ybert J-P. 1975. Emissions polliniques actuelles dans la zone de contact forêtsavane en Côte d'Ivoire. Bull. Soc. Bot. Fr., 122: 251-265.

Ybert J-P. 1979. Atlas des Pollens de Côte d'Ivoire. ORSTOM: Paris; 40p. 